\title{
Variable ventilation from bench to bedside
}

\author{
Robert Huhle ${ }^{1}$, Paolo Pelosi ${ }^{2 *}$ and Marcelo Gama de Abreu ${ }^{1}$
}

\begin{abstract}
This article is one of ten reviews selected from the Annual Update in Intensive Care and Emergency medicine 2016. Other selected articles can be found online at http://www.biomedcentral.com/collections/ annualupdate2016. Further information about the Annual Update in Intensive Care and Emergency Medicine is available from http://www.springer.com/ series/8901.
\end{abstract}

\section{Background}

Biological systems constantly adjust their inner condition according to the external environment in order to achieve a steady state that allows their adaptation to the environment. Healthy biological systems are able to quickly adapt to changing environmental conditions and exhibit intrinsic fluctuations in function within each subsystem, for example the cardiovascular [1] and respiratory [2] systems, during steady-state conditions. In diseased biological systems, however, such intrinsic functional fluctuation (variability) is usually reduced. In fact, reduced variability of the heart rate in patients with coronary heart disease [3], of blood pressure during pre-eclampsia [4], of heart rate and blood pressure during pathological sleep [5], and of respiratory rate and tidal volume in patients with chronic obstructive pulmonary disease (COPD) [6] and prolonged weaning [7] have been documented.

Different from most biological systems, the variability of the respiratory system can be easily influenced in an attempt to improve its function. In controlled, as well as in assisted mechanical ventilation, the variability of tidal volume and/or respiratory rate may be modulated externally by the mechanical ventilator to reproduce certain characteristics of spontaneous breathing in healthy subjects. Because mechanical ventilation represents a common intervention in intensive care and emergency

\footnotetext{
* Correspondence: ppelosi@hotmail.com

University of Genoa, Department of Surgical Sciences and Integrated

Diagnostics, IRCCS AOU San Martino IST, 16131 Genoa, Italy

Full list of author information is available at the end of the article
}

medicine, interest in modes that can enhance the variability of the respiratory pattern has increased in recent years.

In this article, we will review the rationale and mechanisms of variable ventilation, and provide a comprehensive review of the literature for both controlled and assisted variable mechanical ventilation. We will focus mainly on the translational aspects that may be relevant for the clinical practice of mechanical ventilation.

\section{Respiratory variability and rationale}

Physiological breathing patterns are usually highly variable and, to some extent, unpredictable. The variability of a pattern is usually quantified by the coefficient of variation, which is approximately $33 \pm 14.9 \%$ of the tidal volume in healthy spontaneous breathing at rest [8]. The importance of variability in the respiratory pattern is partially explained by the anatomical structure of the lung. The airways and the pulmonary circulation are branching trees with a typical fractal structure in the sense that lower airway generations closely resemble higher generations, and small branches of the pulmonary circulation are similar to larger ones [9]. Such a fractal structure maximizes the area for gas exchange and supports irregular gas-mixing in the lower branches [10]. Breath-by-breath variation in tidal volume and respiratory rate contribute to sustain fast state transition, while minimizing the ratio between tissue stress and strain [11]. Interestingly, tidal volume variability in the physiological resting awake state is generally higher than in other physiological states, as for example during non-rapid eye movement sleep [5] and in the diseased state. In patients with restrictive lung disease [6] and in patients with COPD [12], the variability in the tidal volume pattern is reduced to $22 \pm 5$ and $25.3 \pm 16.3 \%$, respectively.

The basic rationale for variable controlled mechanical ventilation is that the use of a physiological variability in the respiratory pattern, as observed in the healthy resting state, may be beneficial to improve function and reduce damage in the diseased lung. 


\section{Modes of variable ventilation}

For the purposes of this review, a system is considered to be variable/have variability when its input/state/output changes over time. Variability can be regular (or deterministic), irregular, or a combination of the two. Deterministic variability occurs when the output changes in a predictable way, while irregular variability is when the variability follows an unpredictable pattern. Regular variability is usually seen when the pattern of output changes is not complex, as for instance in a sinus wave. Conversely, in irregular variability the pattern of change among levels is complex. For example, tidal breathing has a regular component and an irregular component that changes from cycle to cycle.

Variability of a system can be deterministic (i.e., nonrandom) when it works according to pre-defined rules without random components and the output of the system can be predicted by these rules. Furthermore, systems can show a hybrid or near-deterministic behavior when both deterministic and stochastic components are present, which is usually the case for biological systems. In respect to variable ventilation, patterns with different distributions have been used: Gaussian distribution being purely stochastic and power-law distributions showing hybrid or near-deterministic behavior (Fig. 1). There have been no direct comparisons of the two patterns in an experimental setting, but both show the same effects when compared to non-variable ventilation.

External variation of tidal volume and/or respiratory rate has been used mainly during controlled mechanical ventilation. Since the first description of variable controlled mechanical ventilation by Lefevre et al. [13], various authors have confirmed that variable controlled mechanical ventilation can improve lung mechanics and arterial oxygenation compared to monotonic, regular controlled ventilation in experimental acute lung injury, although this claim has been challenged. Apart from the benefits in gas exchange, the most striking and common finding during variable controlled mechanical ventilation across different studies is the improvement in lung mechanics. In an experimental model of the acute respiratory distress syndrome (ARDS), variable controlled mechanical ventilation improved lung function compared to the ARDS Network lung protective strategy, as well as the open lung approach $[14,15]$. In addition, it has been shown that variable ventilation may also be used during assisted ventilation by means of random variation of pressure support (variable pressure-support ventilation [PSV]) leading to comparable improvements in lung function compared to conventional PSV [16]. Typical signal tracings for flow, volume and airway pressure in variable controlled mechanical ventilation and variable PSV are shown in Fig. 2. Addition of variability to controlled or assisted ventilation modes in general will be referred to as "variable ventilation" throughout this review.

\section{Mechanisms of variable ventilation}

There are two main epiphenomena that underlie improved lung function during variable ventilation:

1. recruitment and stabilization of lung regions contributing to gas exchange; and

2. improvement in ventilation-perfusion matching.

Different macroscopic (respiratory system) and microscopic (alveolar and cell level) mechanisms can explain these epiphenomena (Fig. 3).

Recruitment and stabilization of ventilated lung regions One of the most common problems observed in mechanically ventilated lungs is the closure of peripheral airways

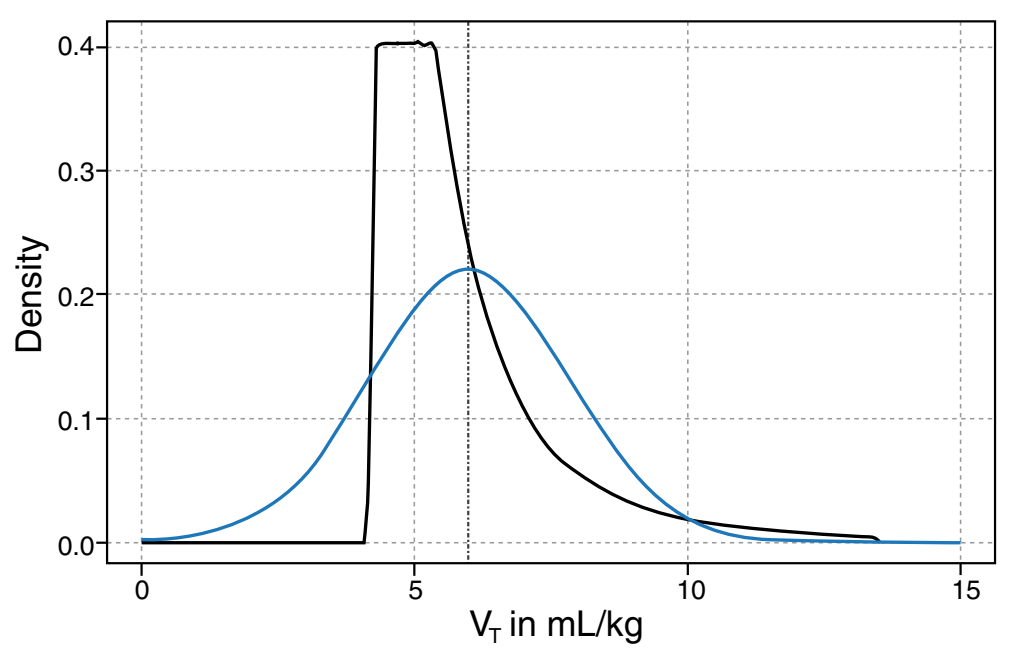

Fig. 1 Distribution of tidal volumes $\left(V_{T}\right)$ according to power-law (black) or Gaussian distribution (blue) 
a

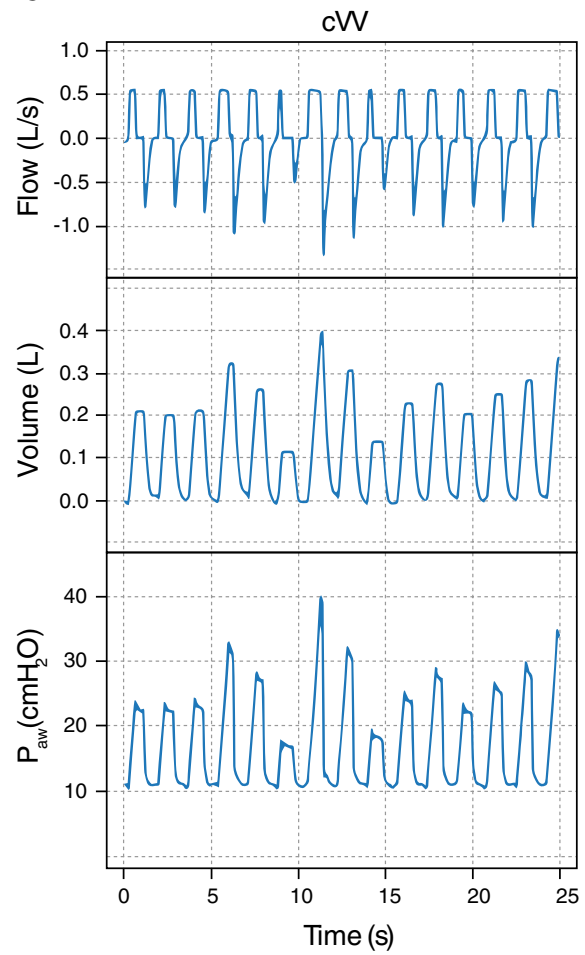

b

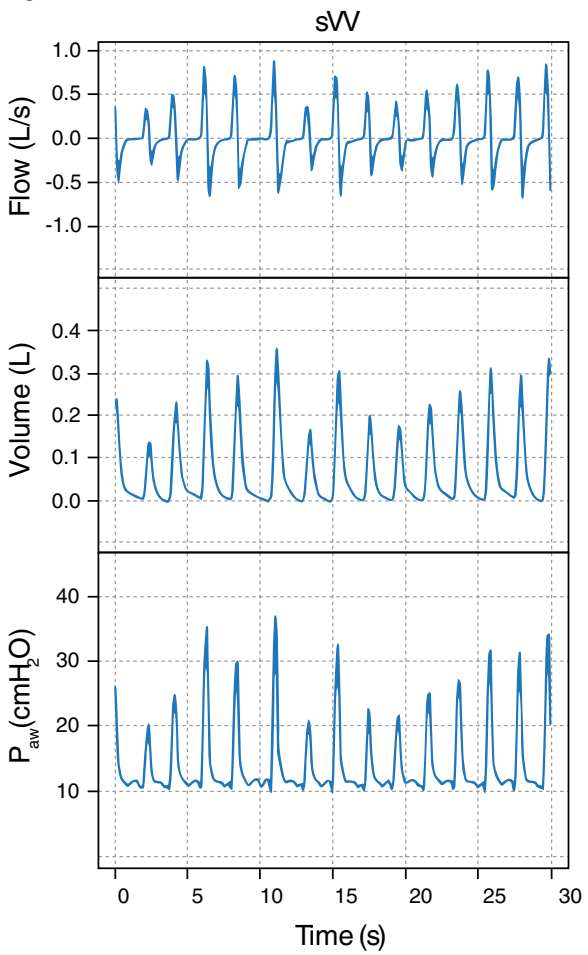

Fig. 2 Signal traces during variable volume controlled ventilation (cWV) and variable pressure support ventilation (sW). $P_{\text {aw }}$ : airway pressure

during expiration. Failure to reopen these airways during inspiration may lead to atelectasis with consequent deterioration of gas exchange and respiratory mechanics. On the other hand, cyclic closing/reopening can increase the shear-stress and trigger the inflammatory response, worsening or leading to lung injury [17].

The beneficial effects of variable ventilation on lung mechanics, especially in acute lung injury, may be explained by the so-called Jensen's theorem [18], which states that on a convex airway pressure versus lung volume relationship (PV curve) the addition of noise to the airway pressure leads to an amplification in the mean tidal volume (also known as Stochastic resonance) [11]. Consequently, during variable ventilation, the driving pressure for a given tidal volume would be theoretically reduced, as shown in Fig. 4. Such a theoretical model, however, also implies that variability could have detrimental effects during mechanical ventilation if high positive end-expiratory pressure (PEEP) is used and/or in more severely injured lungs. In both conditions, ventilation would mainly occur in the zone of the PV curve where variable tidal volumes increase the mean driving pressure. In addition, Jensen's theorem foresees that, in non-injured lungs, variability in tidal volumes would only limit derecruitment, given that no convex portion is present on the PV curve [19].
The amplification of ventilated lung regions is mainly achieved by recruitment of previously collapsed alveoli. Suki et al. [20] showed that once the critical opening pressure $\left(\mathrm{P}_{\text {crit }}\right)$ of collapsed airways/alveoli has been exceeded, all subtended or daughter airways/alveoli with lower $\mathrm{P}_{\text {crit }}$ will be opened like an avalanche. Since the $\mathrm{P}_{\text {crit }}$ values of closed airways as well as the time to achieve those values may differ across the lungs, mechanical ventilation patterns that produce different airway pressures and inspiratory times may be advantageous to maximize lung recruitment and stabilization, as compared to regular patterns.

For stabilization of these newly opened lung regions and prevention of collapse during mechanical ventilation of healthy lungs, the production and release of surfactant is crucial [21]. Surfactant release increases exponentially with stretch in alveolar type II cells. Thus, high tidal volume cycles during variable ventilation may stimulate the release of surfactant to a similar or even higher degree than the average increased stretch during conventional mechanical ventilation [22].

\section{Enhanced ventilation-perfusion matching}

During variable ventilation, improvement in gas exchange is usually a consequence of enhanced ventilation/perfusion (V/Q) matching, which results either from redistribution 


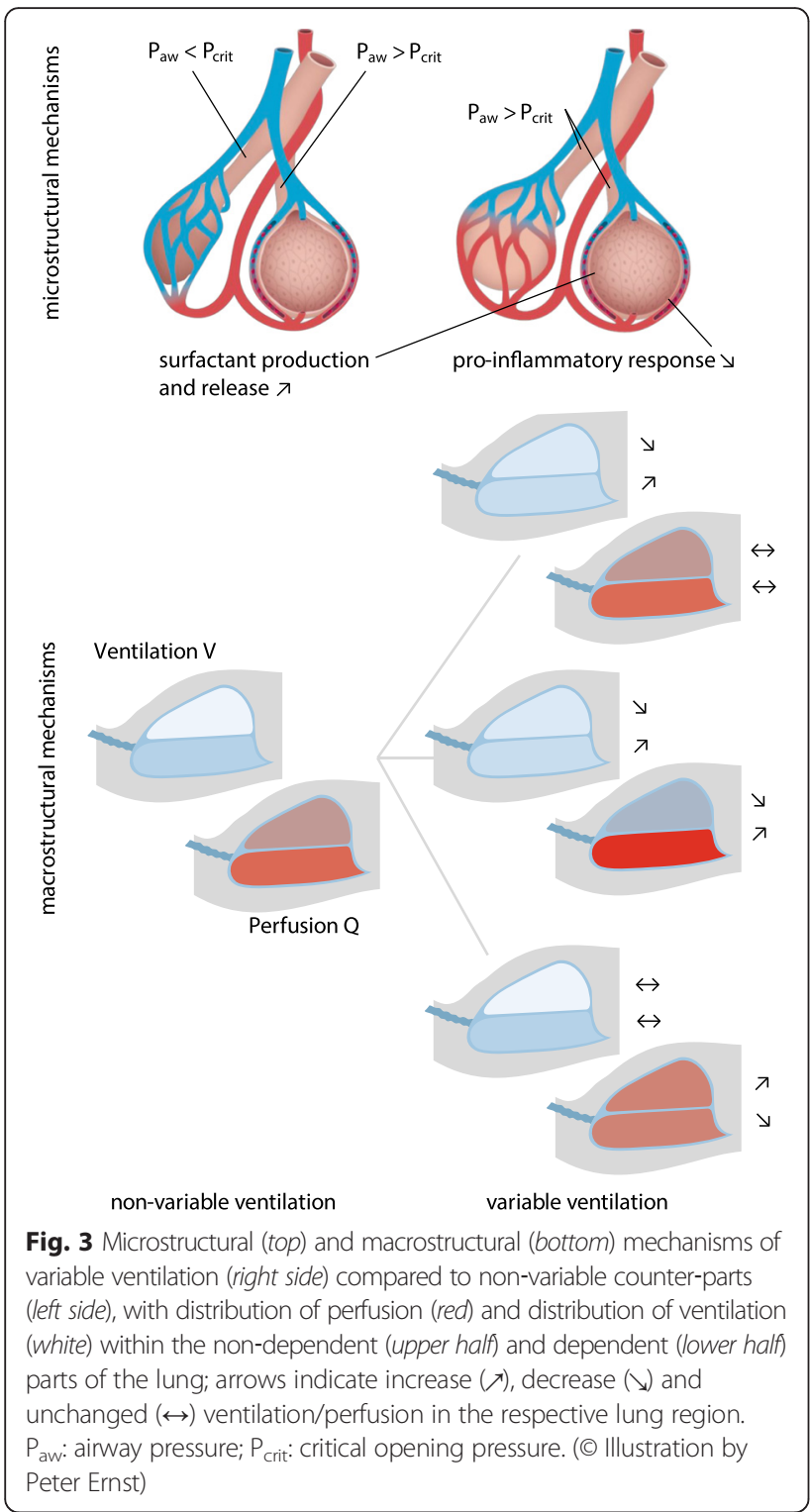

of ventilation to perfused areas (reduction of shunt), or redistribution of pulmonary blood flow towards better ventilated lung zones (decreasing dead space). Variable ventilation leads intermittently to airway pressures that exceed the critical opening pressures of single airways in dependent lung zones, resulting in alveolar recruitment in those areas. Following that, aeration and ventilation increase in the dependent lung and, as perfusion shows a prominent gravity-dependent ventral-to-caudal gradient, local and global V/Q matching increase. Perfusion may also accompany the redistribution of ventilation to dependent lung zones, further increasing the $\mathrm{V} / \mathrm{Q}$ matching.

However, gas exchange may also improve without redistribution of ventilation. It has been suggested that during variable PSV, oxygenation increases despite a lack of improvement of aeration in dependent lung zones. In fact, in experimental models of ARDS, a redistribution of perfusion from dependent to non-dependent lung regions has been observed [23], resulting in enhanced V/Q matching. Therefore, in the presence of preserved hypoxic pulmonary vasoconstriction, a phenomenon of 'capillary recruitment' may occur, shifting perfusion towards the better aerated and ventilated non-dependent lung zones.

\section{Variable mechanical ventilation in experimental studies (Table 1)}

\section{Healthy and pre-term lungs}

In anesthetized pigs without lung injury, variable controlled mechanical ventilation compared to conventional controlled mechanical ventilation prevented the deterioration in gas exchange that is usually observed during prolonged mechanical ventilation [24].

In pre-term lambs with immature lungs, variable controlled mechanical ventilation compared to conventional controlled mechanical ventilation improved the dynamic respiratory system compliance and reduced the $\mathrm{PaCO}_{2}$ without influencing oxygenation, the protein content in the bronchoalveolar lavage (BAL) fluid, or the gene expression of interleukin (IL)-1 $\beta$ [25].

Similarly, respiratory system elastance increased during conventional mechanical ventilation but to a smaller amount during variable ventilation without significant effects on ventral-dorsal and craniocaudal reduction of aeration or lung tissue cytokine concentrations [26, 27].

\section{Models of acute respiratory distress syndrome}

Most investigations on variable ventilation have been performed in experimental models of ARDS using different animal species (86 \% in large animals and $14 \%$ in rodents) and injury models (50\% oleic acid aspiration, $35 \%$ surfactant depletion, $10 \%$ hydrochloric $(\mathrm{HCl})$ acid aspiration). However, ARDS models do not reproduce all features of human ARDS. Moreover, the degrees of recruitability, tissue damage and inflammation differ widely among models. Although the saline lung lavage model usually shows the best recruitability and relatively low inflammatory response, $\mathrm{HCl}$ acid aspiration typically results in a more heterogeneous injury and less recruitable lung. In this section, the main results of the numerous experimental studies are discussed.

\section{Gas exchange effects}

Variable controlled mechanical ventilation outperformed conventional controlled mechanical ventilation in terms of arterial oxygenation in 10 out of 12 experimental studies in models of ARDS, including surfactant depletion [15, 28], oleic acid [13, 14, 29-31] and $\mathrm{HCl}$ aspiration [32]. In two studies using oleic acid injury, variable 


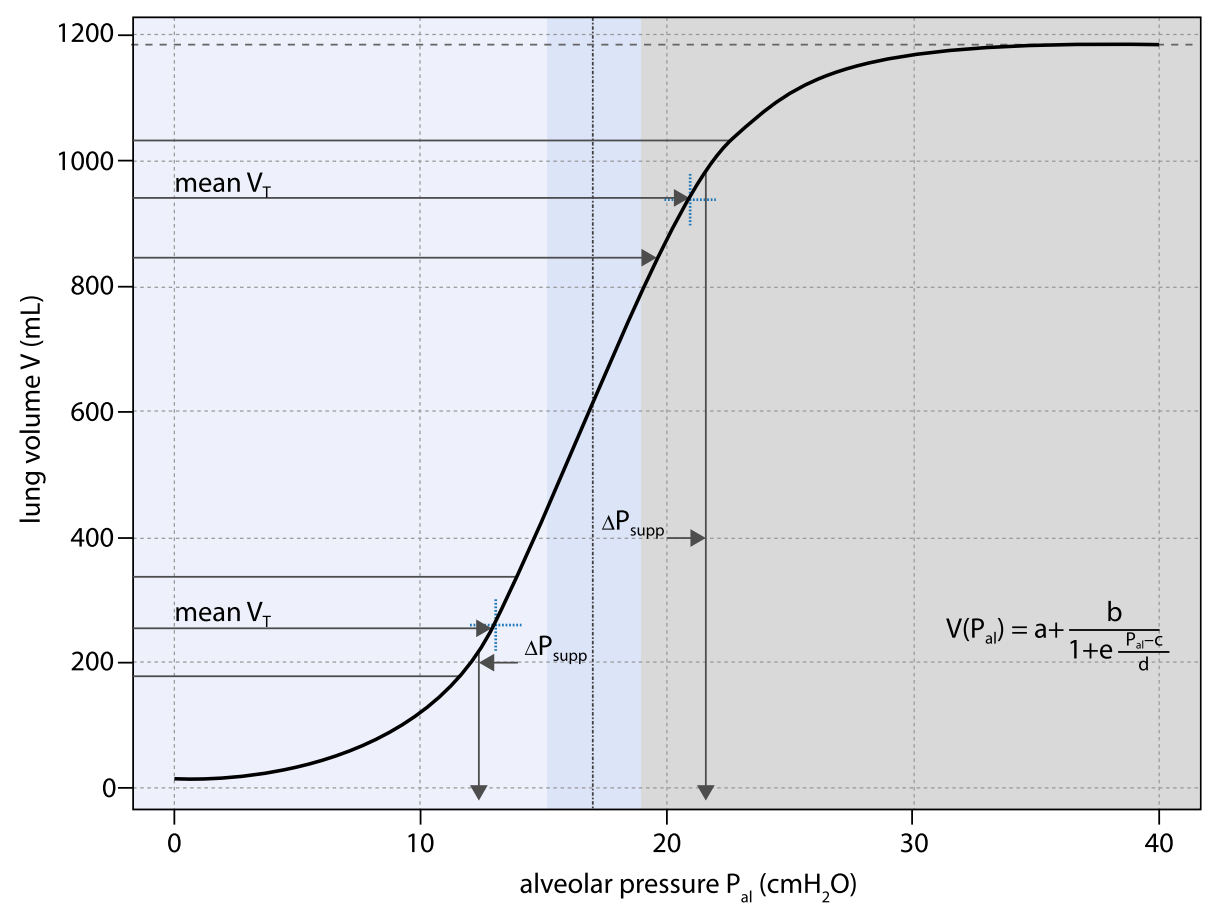

Fig. 4 Advantageous (blue) and disadvantageous (gray) ranges of alveolar pressure of variable ventilation on a representative static pressure - volume curve described by the Venegas equation with parameters $\mathrm{a}, \mathrm{b}, \mathrm{c}$ and $\mathrm{d}$ from [18]. $\Delta \mathrm{P}_{\text {supp: }}$ : change in support pressure needed to gain the displayed tidal volume $\left(\mathrm{V}_{T}\right)$ in variable ventilation

controlled mechanical ventilation did not improve arterial oxygenation compared to conventional controlled mechanical ventilation in mongrel dogs [33] or in pigs [34]. Interestingly, the effects of variable ventilation on arterial oxygenation seem to be dependent on the degree of tidal volume variability. In guinea pigs with lung injury induced by endotoxin, a coefficient of variation of $23-35 \%$ in tidal volume maximized arterial oxygenation [35].

Improvements in gas exchange were also shown during variable PSV compared to conventional PSV in pigs with lung injury induced by saline lung lavage [16, 23, 36, 37]. Such effects seem also to depend on the degree of variability of pressure support variability, whereby a coefficient of variation of $30 \%$ was associated with the highest levels of arterial oxygenation [37] while for a coefficient of variation $>30 \%$ detrimental effects on hemodynamics inhibited a further increase in $\mathrm{PaO}_{2}$.

Independent of the injury model and also in lung healthy animals, the $\mathrm{PaCO}_{2}$ was reduced during variable ventilation compared to conventional mechanical ventilation when minute ventilation was comparable between groups [23, 24, 28, 38].

\section{Ventilation-perfusion matching}

In an oleic acid model of ARDS in pigs, total lung volume measured by computed tomography (CT) increased after $4 \mathrm{~h}$ of variable ventilation but not with conventional mechanical ventilation [31, 34]. Variable ventilation also resulted in a significant increase in normally aerated, and a decrease in non- and poorly aerated lung tissue. In addition, with variable controlled mechanical ventilation compared to conventional controlled mechanical ventilation, surfactant was simultaneously redistributed towards dorsal regions. Thus, although perfusion was not measured and the regions of recruitment were not reported, an increase in V/Q matching was indicated. Assisted variable ventilation had no effects on recruitment or on redistribution of aeration in a surfactant depletion model when compared to conventional assisted ventilation $[16,39]$.

In porcine models of lung injury induced by oleic acid $[13,14,29-31,40]$ and surfactant depletion [15], as well as in healthy lungs [24], variable controlled mechanical ventilation reduced pulmonary shunt. Similarly, venous admixture was reduced in variable PSV but not in conventional PSV $[16,36]$. In a model of lung injury induced by oleic acid in pigs $[13,14,34]$, variable controlled mechanical ventilation did not importantly influence the dead space, suggesting that shunt reduction is more prominent then reduction of dead space during variable ventilation.

\section{Lung mechanics}

In 10 out of 12 experimental studies [13-15, 27-35] in different species and models of ARDS, respiratory system compliance $\left(C_{R S}\right)$ was positively influenced by variable 
Table 1 Effects of variable ventilation compared to conventional ventilation from in vitro and in vivo experimental models

\begin{tabular}{|c|c|c|c|c|c|}
\hline Endpoint & Effect & Species & Disease model & Type of variable ventilation mode & References \\
\hline \multirow[t]{10}{*}{ Oxygenation } & $\nearrow$ & Porcine/ & Healthy & Controlled & [24] \\
\hline & $\nearrow$ & canine & ARDS (surfactant lavage) & Controlled/assisted & {$[15,16,28]$} \\
\hline & $\nearrow$ & & ARDS (oleic acid) & Controlled & {$[13,14,29,30]$} \\
\hline & $\nearrow$ & & ARDS (double-hit model') & Controlled/assisted & {$[36,50]$} \\
\hline & $\leftrightarrow$ & & ARDS (oleic acid) & Controlled & {$[31,33,34]$} \\
\hline & $\nearrow$ & Guinea pig & ARDS (endotoxin induced) & Controlled & [35] \\
\hline & $\nearrow$ & & Healthy & Controlled & {$[38]$} \\
\hline & $\leftrightarrow$ & Rats & Healthy & Controlled & {$[26]$} \\
\hline & $\nearrow$ & & ARDS ( $\mathrm{HCl}$ acid) & Controlled & {$[27]$} \\
\hline & $\nearrow$ & Mice & ARDS ( $\mathrm{HCl}$ acid) & Controlled & {$[32]$} \\
\hline \multirow[t]{2}{*}{ Aeration } & $\nearrow$ & Porcine & ARDS (oleic acid) & Controlled & {$[31]$} \\
\hline & $\leftrightarrow$ & & ARDS (surfactant lavage) & Assisted & [39] \\
\hline Perfusion & $\nearrow$ & Porcine & ARDS (surfactant lavage) & Assisted & [39] \\
\hline \multirow{9}{*}{$\begin{array}{l}\text { Respiratory system } \\
\text { compliance }\left(C_{R S}\right)\end{array}$} & $\nearrow$ & Porcine/ & Healthy & Controlled & {$[24]$} \\
\hline & $\nearrow$ & canine & ARDS (surfactant lavage) & Controlled/assisted & {$[15,16,28]$} \\
\hline & $\nearrow$ & & ARDS (oleic acid) & Controlled & {$[13,29-31,34]$} \\
\hline & $\leftrightarrow$ & & & & {$[14,33]$} \\
\hline & $\nearrow$ & & ARDS (double-hit model') & Controlled/assisted & {$[36,50]$} \\
\hline & $\nearrow$ & Guinea pig & ARDS (endotoxin induced) & Controlled & {$[35]$} \\
\hline & $\leftrightarrow$ & & Healthy & & {$[38]$} \\
\hline & $\nearrow$ & Rats & Healthy; ARDS ( $\mathrm{HCl}$ acid) & Controlled & {$[26,27]$} \\
\hline & $\nearrow$ & Mice & ARDS ( $\mathrm{HCl}$ acid) & Controlled & {$[32]$} \\
\hline \multirow{2}{*}{$\begin{array}{l}\text { Surfactant release } \\
\text { and production }\end{array}$} & $\nearrow$ & Guinea pig & None & Controlled & {$[38]$} \\
\hline & $\leftrightarrow$ & Porcine & ARDS (oleic acid) & Controlled & [30] \\
\hline \multirow[t]{7}{*}{ Inflammation } & $\searrow$ & Cell & ARDS (LPS) & Controlled & {$[43]$} \\
\hline & $\searrow$ & Mice & ARDS ( $\mathrm{HCl}$ acid) & Controlled & {$[32]$} \\
\hline & $\searrow$ & Guinea pig & None & Controlled & {$[38]$} \\
\hline & $\searrow$ & Porcine & ARDS (oleic acid) & Controlled & [14] \\
\hline & $\leftrightarrow$ & & ARDS (oleic acid/surfactant lavage); Bronchospasm & Controlled & {$[15,30,42]$} \\
\hline & $\leftrightarrow$ & Lambs & Pre-term & Controlled & {$[25]$} \\
\hline & $\leftrightarrow$ & Porcine & ARDS (surfactant lavage) & Assisted & {$[23,36]$} \\
\hline \multirow[t]{3}{*}{ Damage (histology) } & $\searrow$ & Porcine & ARDS (surfactant lavage) & Controlled & {$[15]$} \\
\hline & $\leftrightarrow$ & & ARDS (oleic acid) & & {$[30]$} \\
\hline & $\leftrightarrow$ & & ARDS (surfactant lavage) & Assisted & {$[23,36]$} \\
\hline \multirow[t]{2}{*}{ Resolution of edema } & $\lambda_{\leftrightarrow}$ & Porcine & ARDS (oleic acid) & Controlled & {$[34]$} \\
\hline & $\nearrow$ & & ARDS (surfactant lavage) & Assisted & {$[23,36]$} \\
\hline
\end{tabular}

controlled mechanical ventilation. There are only two studies, in a porcine oleic acid injury model, in which $\mathrm{C}_{\mathrm{RS}}$ was not affected positively by variable ventilation $[14,33] . C_{R S}$ showed a linear dependence on the level of variability, reaching its maximal values at a coefficient of variation $=35 \%$ [35]. Respiratory system resistance $\left(R_{R S}\right)$ was only improved by variable controlled mechanical ventilation in one [32] of three studies [26, $27,32]$ in a rodent acid aspiration model. However, an effect on $R_{R S}$ may only be secondary to recruitment of large portions of the lung (see section on "Mechanisms of variable ventilation" above).

In a porcine surfactant depletion model, variable PSV improved lung mechanics, reducing $R_{R S}[36$ ] and increasing $C_{R S}[16,23,36,37,39,41]$. Comparable $C_{R S}$ improved linearly with variability of the support, reaching its maximum at a coefficient of variation of $45 \%$ [37]. 


\section{Surfactant production and release}

In healthy guinea pigs, variable controlled mechanical ventilation led to an increase in surfactant-associated phospholipid concentration and a decrease in membrane-associated phospholipid concentration compared to conventional controlled mechanical ventilation [38]. In contrast, in oleic acid-injured pigs, variable controlled mechanical ventilation had no positive effects on surfactant surface tension as measured by capillary surfactometry on raw and chloroform/methanol extracted BAL fluid [30].

In non-ARDS lungs, variable controlled mechanical ventilation augmented surfactant secretion, but had no effect on surfactant surface tension in oleic acid injured lungs. Thus, in healthy lungs, but not in injured lungs, surfactant production and release might be an important mechanism to explain the benefits of variable ventilation. However further investigations in different models of experimental ARDS are necessary.

\section{Inflammation and damage}

Inflammation in the lung occurs during ARDS as a consequence of cell injury. Depending on the ventilator strategy, the initial injury may be amplified, mediating pulmonary edema, alveolar disruption and release of cytokines [17]. Thus, a potential means of quantifying the protectiveness of a ventilation mode is the measurement of inflammatory cytokine and mRNA concentrations in BAL fluid and lung tissue, respectively.

In tracheal aspirates from oleic acid-injured pigs, IL-8 concentrations decreased after $5 \mathrm{~h}$ of variable controlled mechanical ventilation compared to conventional controlled mechanical ventilation but the investigated pro-inflammatory cytokines, tumor necrosis factor- $\alpha$ (TNF- $\alpha$ ) and IL-6, were not measurable in serum or in tracheal aspirates. The concentration of the anti-inflammatory cytokine, IL-10, did not differ between groups in serum or tracheal aspirate samples. In $\mathrm{HCl}$-injured mice, IL-1 $\beta$ levels, as measured by Western Blot Analysis, were significantly higher with conventional controlled mechanical ventilation than in the baseline injury group and with variable ventilation [32]. In guinea pigs after $3 \mathrm{~h}$ of variable controlled mechanical ventilation, similar results were seen in the concentrations of the pro-inflammatory cytokines TNF- $\alpha$, IL- 6 and monocyte chemoattractant protein-1 (MCP-1) in BAL fluid [38]. TNF- $\alpha$ concentrations were two-fold and tenfold increased during conventional controlled mechanical ventilation compared with variable controlled mechanical ventilation and unventilated controls, respectively. IL-6 concentrations were increased six-fold and thirty fold in conventional controlled mechanical ventilation compared to variable controlled mechanical ventilation and unventilated controls, respectively, and similarly MCP-1 was increased about three-fold and six-fold in conventional controlled mechanical ventilation compared to variable controlled mechanical ventilation and unventilated controls, respectively.

By contrast, several groups found no differences in the inflammatory response between conventional and variable controlled mechanical ventilation. In an oleic acid injured porcine model, IL-8 concentrations in BAL fluid were similar with conventional and variable controlled mechanical ventilation [30]. In a model of bronchospasm initiated by administration of methacholine aerosol in pigs, there were no differences in the concentrations of IL- 6 and IL10 in BAL fluid with variable compared to conventional controlled mechanical ventilation [42]. In preterm-lambs, there were no differences between conventional and variable controlled mechanical ventilation in total protein content for BAL fluid and mRNA levels of IL1- $\beta$ or quantitative reverse transcription polymerase chain reaction (qRT-PCR) in lung tissue [25].

In a surfactant depletion model in pigs, gene expression and lung tissue cytokine levels of IL-6, IL-8 and transforming growth factor- $\beta$ (TGF- $\beta$ ) were not different between variable controlled mechanical ventilation and conventional controlled mechanical ventilation after $6 \mathrm{~h}$ of therapy [15]. Similarly, in the same injury model, no effects of variable PSV were found on plasma or tissue levels of inflammatory markers compared to PSV $[23,36]$.

The available literature is not conclusive on the relationship between inflammation and variable controlled mechanical ventilation compared to conventional controlled mechanical ventilation. Although in studies in large animals no differences in pro-inflammatory or anti-inflammatory reactions could be established during ARDS independent of the animal model, studies performed on healthy and $\mathrm{HCl}$-injured rodents suggest a reduced pro-inflammatory response during variable ventilation. However, an in vitro study on lipopolysaccharide (LPS)-injured alveolar epithelial L2-cells confirmed a reduction in the inflammatory response, involving the ERK1/2 signaling pathway, suggesting a cellular mechanism, when a variable instead of a constant tidal stretch was applied [43].

There were no differences in lung injury in post-mortem acquired tissue samples of the lung with variable controlled mechanical ventilation compared to conventional controlled mechanical ventilation in oleic acid injury [30], but in a surfactant depletion model variable controlled mechanical ventilation reduced overall tissue damage as assessed by the diffuse alveolar damage (DAD) score when compared to conventional controlled mechanical ventilation [15] and variable controlled mechanical ventilation was also associated with reduced interstitial edema, hemorrhage and epithelial dysfunction in this study. In a porcine oleic acid model, total lung weight and density were reduced with variable controlled mechanical ventilation compared to conventional controlled mechanical 
ventilation and average alveolar fluid clearance rates were positive compared to negative [34]. However, analysis of corresponding wet:dry ratios of the post-mortem lung showed no effects of variable controlled mechanical ventilation compared to conventional controlled mechanical ventilation in five out of six investigations, independent of the injury model $[13,29-31,34,44]$. Consequently, variable controlled mechanical ventilation has only limited effects on edema clearance during ARDS independent of ventilator mode. Comparably, in a surfactant depletion model in pigs, alveolar edema was slightly but significantly reduced in variable PSV compared to conventional PSV $[23,36]$.

\section{Non-ARDS models}

Variable controlled mechanical ventilation has also been reported to improve lung function in porcine models of severe bronchospasm [42], atelectasis [40] and one-lung ventilation [44]. In each of these models variable controlled mechanical ventilation was associated with increased $\mathrm{PaO}_{2}$ and $\mathrm{C}_{\mathrm{RS}}$ and decreased $\mathrm{PaCO}_{2}$ compared to conventional controlled mechanical ventilation. Additionally shunt fraction was reduced by variable controlled mechanical ventilation in the one-lung ventilation and atelectasis models [40,44], dead space was reduced only in the one-lung ventilation model [44] and $R_{R S}$ only in the asthma model [42]. The results of the study in severe bronchospasm [42] are of special importance as they show that variable controlled mechanical ventilation can improve lung function also in models of secondary atelectasis and can also reduce $R_{R S}$. This finding can be explained by the excessive critical opening pressures that are potentially even higher in this asthma model than in ARDS models.

\section{Clinical application of variable mechanical ventilation}

Variable ventilation has been studied in only a small number of clinical trials to date, which are summarized below.

\section{Variable controlled mechanical ventilation}

Variable controlled mechanical ventilation was first used in a clinical setting by Boker et al. [45] in an open-label, randomized, two-arm, longitudinal perioperative study. Forty-one patients undergoing abdominal aortic aneurysmectomy were ventilated with conventional or variable controlled mechanical ventilation for $6 \mathrm{~h}$ during surgery and subsequent recovery. Exclusion criteria were chronic diseases of respiratory and cardiovascular systems, obesity (body mass index $[\mathrm{BMI}]>35 \mathrm{~kg} / \mathrm{m}^{2}$ ), previous thoracic surgery, drug abuse or pregnancy. Anesthesia consisted of propofol, sufentanil and rocuronium, initiated by intravenous bolus doses and infused continuously for $6 \mathrm{~h}$ with an additional continuous infusion of $0.06 \mathrm{mg} / \mathrm{ml}$ bupivacaine and $30 \mathrm{mcg} / \mathrm{ml}$ hydromorphone for pain control. Initially ventilator settings were tidal volume $10 \mathrm{ml} / \mathrm{kg}$ (ideal body weight), respiratory rate $10 \mathrm{breath} / \mathrm{min}$ (adjusted for a $\mathrm{PaCO}_{2}$ target range $35-45 \mathrm{mmHg}$ ), zero positive endinspiratory pressure, I:E ratio of $1: 2$ and $\mathrm{FiO}_{2}$ of 0.6. Respiratory mechanics, blood gas and hemodynamics were monitored, analyzed offline and compared. A significant group-time interaction was found for $\mathrm{PaO}_{2}$ between groups, being increased by approximately $40 \mathrm{mmHg}$ with variable controlled mechanical ventilation at 3,4 and $5 \mathrm{~h}$ of therapy. $\mathrm{PaCO}_{2}$ did not differ between groups as intended by the protocol but the minute ventilation to accomplish this goal was significantly reduced in the variable controlled mechanical ventilation group $(8.1 \pm 1$ vs. $7.7 \pm 1.1 \mathrm{l} / \mathrm{min}$ ). Dead space ventilation was significantly reduced with variable controlled mechanical ventilation compared to conventional controlled mechanical ventilation (group $\mathrm{x}$ time effects). Static compliance was significantly increased in the variable ventilation group after $3 \mathrm{~h}$ $\left(0.54 \pm 0.13\right.$ vs. $\left.0.62 \pm 0.17 \mathrm{ml} / \mathrm{cmH}_{2} \mathrm{O} / \mathrm{kg}\right)$. This study suggested that variable controlled mechanical ventilation was advantageous for lung function even in healthy lungs during abdominal surgery.

In a pilot cross-over study in eight critically ill patients ventilated for at least $72 \mathrm{~h}$ in a postsurgical ICU, oxygen index $\left(\mathrm{PaO}_{2}=7.1\right.$ vs. $\left.11.5 \mathrm{cmH}_{2} \mathrm{O} / \mathrm{mmHg}, \mathrm{p}=0.034\right)$ and static lung compliance $\left(0.36\right.$ vs. $0.34 \mathrm{ml} / \mathrm{cmH}_{2} \mathrm{O} / \mathrm{kg}$, $\mathrm{p}=0.049)$ were increased and dead space decreased (0.64 vs. $0.68, p=0.017$ ) during variable compared with conventional controlled mechanical ventilation. $\mathrm{PaCO}_{2}$ did not differ between the modes of ventilation [46]. Patients had a baseline Horovitz Index of $100-300 \mathrm{mmHg}$ and were randomly selected to begin either with conventional or with variable controlled mechanical ventilation for four hour periods for each mode. Tidal volume was set to $6 \mathrm{ml} / \mathrm{kg}$ to comply with the ARDSnet protocol. Respiratory rate and PEEP remained constant. Unfortunately patient anamneses as well as methods to obtain static lung compliance were not reported in detail.

Although the first theoretical framework suggested increased benefits of variable ventilation in damaged lungs with large collapsed and recruitable regions, there are no large study population data available on variable ventilation in a clinical setting in ARDS patients. Clinical studies are currently in progress using variable controlled mechanical ventilation during open-abdominal surgery [47] and in patients with ARDS (ClinicalTrials.gov ID: NCT00202098 and NCT01083277).

\section{Variable assisted mechanical ventilation}

In a randomized crossover study performed in $13 \mathrm{ICU}$ patients with acute hypoxemic respiratory failure, variable PSV was not associated with adverse events. Compared to 
traditional PSV, variable PSV yielded no differences in gas exchange, hemodynamics, or lung mechanics. The reported increase in tidal volume variability $(24.4 \pm 7.8 \%$ vs. $13.7 \pm 9.1 \%$ ) during variable PSV was associated with improved patient-ventilator synchrony. The externally increased tidal volume variability mimics the intrinsic healthy variability more closely than can be achieved by the patient through simple on/off triggering during conventional PSV, which may explain the improved patientventilator synchrony [48].

Clinical studies using variable PSV during weaning [49], respiratory failure (ClinicalTrials.gov ID: NCT02499276 and NCT01580956) and ARDS (NCT00267241) are being conducted. The results of these investigations will shed further light into the potential applications of this ventilation strategy.

\section{Potential application of variable ventilation}

Variable ventilation is probably one of the ventilatory strategies that has undergone most extensive testing in animal models of disease, as well as in small patient series, before being introduced into clinical practice. Such studies have consistently showed that variable controlled mechanical ventilation improves lung function, and reduces or does not worsen lung damage and inflammation compared to non-variable modes. The most promising potential of variable controlled mechanical ventilation is to decrease and prevent deterioration of the mean driving pressure during mechanical ventilation. However, experience with variable controlled mechanical ventilation is so far limited to mild and moderate lung injury, as well as relatively short periods of time. Furthermore, variable controlled mechanical ventilation is not yet commercially available, precluding its clinical use.

In contrast to variable controlled mechanical ventilation, variable PSV is available for clinical use, and preliminary results in small patient series indicate it may improve patient/ventilator synchrony, although its effects on lung function are not as pronounced as those of variable controlled mechanical ventilation. Possible clinical applications of variable PSV include: reduction in the inspiratory work of breathing in patients with increased respiratory drive; improvement in patient/ventilator synchrony in the presence of restrictive lung disease; increase in the variability of the respiratory pattern in patients with reduced intrinsic variability; weaning from the mechanical ventilator.

\section{Conclusion}

Variable ventilation enables some aspects of the respiratory pattern of healthy, spontaneously breathing subjects to be mimicked in mechanically ventilated patients. Experimental studies have shown that variable controlled mechanical ventilation improves lung function and reduces damage in mild to moderate lung injury, in the short term. Similar, but less pronounced findings have been reported with variable pressure support ventilation in models of acute lung injury. Initial clinical experience suggests that both variable controlled and variable support ventilation can be safely applied, but not necessarily with improved lung function. Variable PSV is potentially associated with improved patient/ventilator synchrony. Ongoing clinical studies on variable ventilation may contribute to define the role of variable ventilation in the ICU and emergency room.

\section{Competing interests}

M. Gama de Abreu was granted a patent on the variable pressure support ventilation mode of assisted ventilation, which has been licensed to Dräger Medical AG (Lübeck, Germany).

Authors' contributions

All authors read and approved the final manuscript.

\section{Declarations}

Publication of this article was funded by Deutsche Forschungsgemeinschaft Grant number: GA1256/7-1.

\section{Author details}

${ }^{1}$ Pulmonary Engineering Group, Department of Anaesthesiology and Intensive Care Medicine, University Hospital Carl Gustav Carus, Technische Universität Dresden, Dresden, Germany. ${ }^{2}$ University of Genoa, Department of Surgical Sciences and Integrated Diagnostics, IRCCS AOU San Martino IST, 16131 Genoa, Italy.

Published online: 15 March 2016

\section{References}

1. Ivanov $P C$, Amaral $L A$, Goldberger $A L$, et al. Multifractality in human heartbeat dynamics. Nature. 1999;399:461-5.

2. Frey U, Silverman M, Barabási AL, Suki B. Irregularities and power law distributions in the breathing pattern in preterm and term infants. J Appl Physiol Bethesda Md. 1998;1985(85):789-97.

3. Huikuri HV, Mäkikallio TH. Heart rate variability in ischemic heart disease. Auton Neurosci Basic Clin. 2001:90:95-101.

4. Malberg H, Bauernschmitt R, Voss A, et al. Analysis of cardiovascular oscillations: a new approach to the early prediction of pre-eclampsia. Chaos. 2007;17:015113.

5. Penzel T, Wessel N, Riedl M, et al. Cardiovascular and respiratory dynamics during normal and pathological sleep. Chaos. 2007;17:015116.

6. Brack T, Jubran A, Tobin MJ. Dyspnea and decreased variability of breathing in patients with restrictive lung disease. Am J Respir Crit Care Med. 2002;165:1260-4.

7. Wysocki M, Cracco C, Teixeira A, et al. Reduced breathing variability as a predictor of unsuccessful patient separation from mechanical ventilation. Crit Care Med. 2006:34:2076-83.

8. Tobin MJ, Mador MJ, Guenther SM, Lodato RF, Sackner MA. Variability of resting respiratory drive and timing in healthy subjects. J Appl Physiol. 1988;1985(65):309-17.

9. Boxt LM, Katz J, Liebovitch LS, Jones R, Esser PD, Reid L. Fractal analysis of pulmonary arteries: the fractal dimension is lower in pulmonary hypertension. J Thorac Imaging. 1994;9:8-13.

10. Tsuda A, Rogers RA, Hydon PE, Butler JP. Chaotic mixing deep in the lung Proc Natl Acad Sci U S A. 2002:99:10173-8.

11. Suki B, Alencar AM, Sujeer MK, et al. Life-support system benefits from noise Nature. 1998:393:127-8.

12. Loveridge $B$, West $P$, Anthonisen NR, Kryger $M H$. Breathing patterns in patients with chronic obstructive pulmonary disease. Am Rev Respir Dis. 1984;130:730-3.

13. Lefevre GR, Kowalski SE, Girling LG, Thiessen DB, Mutch WA. Improved arterial oxygenation after oleic acid lung injury in the pig using a computer-controlled mechanical ventilator. Am J Respir Crit Care Med. 1996;154:1567-72. 
14. Boker A, Graham MR, Walley KR, et al. Improved arterial oxygenation with biologically variable or fractal ventilation using low tidal volumes in a porcine model of acute respiratory distress syndrome. Am J Respir Crit Care Med. 2002;165:456-62.

15. Spieth PM, Carvalho AR, Pelosi $P$, et al. Variable tidal volumes improve lung protective ventilation strategies in experimental lung injury. Am J Respir Crit Care Med. 2009;179:684-93.

16. de Gama AM, Spieth PM, Pelosi P, et al. Noisy pressure support ventilation: a pilot study on a new assisted ventilation mode in experimental lung injury. Crit Care Med. 2008;36:818-27.

17. Slutsky AS, Ranieri VM. Ventilator-induced lung injury. N Engl J Med. 2013; 369:2126-36.

18. Venegas JG, Harris RS, Simon BA. A comprehensive equation for the pulmonary pressure-volume curve. J Appl Physiol Bethesda Md. 1998; 1985(84):389-95.

19. Runck H, Schumann S, Tacke S, Haberstroh J, Guttmann J. Time-dependent recruitment effects in ventilated healthy and lung-injured rats: "recruitmentmemory". Respir Physiol Neurobiol. 2012;184:65-72.

20. Suki B, Barabási AL, Hantos Z, Peták F, Stanley HE. Avalanches and powerlaw behaviour in lung inflation. Nature. 1994;368:615-8.

21. Lutz D, Gazdhar A, Lopez-Rodriguez E, et al. Alveolar derecruitment and collapse induration as crucial mechanisms in lung injury and fibrosis. Am J Respir Cell Mol Biol. 2015;52:232-43.

22. Wirtz HR, Dobbs LG. Calcium mobilization and exocytosis after one mechanical stretch of lung epithelial cells. Science. 1990;250:1266-9.

23. Spieth PM, Carvalho AR, Güldner A, et al. Pressure support improves oxygenation and lung protection compared to pressure-controlled ventilation and is further improved by random variation of pressure support. Crit Care Med. 2011;39:746-55.

24. Mutch WA, Eschun GM, Kowalski SE, Graham MR, Girling LG, Lefevre GR. Biologically variable ventilation prevents deterioration of gas exchange during prolonged anaesthesia. Br J Anaesth. 2000;84:197-203.

25. Pillow JJ, Musk GC, McLean CM, et al. Variable ventilation improves ventilation and lung compliance in preterm lambs. Intensive Care Med. 2011;37:1352-9.

26. Camilo LM, Ávila MB, Cruz LFS, et al. Positive end-expiratory pressure and variable ventilation in lung-healthy rats under general anesthesia. PLOS ONE. 2014;9:e110817.

27. Spieth PM, Bluth T, Hegeman MA, et al. Mechanical ventilation with variable tidal volumes in a rodent model of acute acid aspiration. Am J Respir Crit care Med Meeting Abstracts. 2013;187:A1118 (abst).

28. Bellardine $\mathrm{CL}$, Hoffman AM, Tsai L, et al. Comparison of variable and conventional ventilation in a sheep saline lavage lung injury model. Crit Care Med. 2006;34:439-45.

29. Mutch WA, Harms S, Lefevre GR, Graham MR, Girling LG, Kowalski SE. Biologically variable ventilation increases arterial oxygenation over that seen with positive end-expiratory pressure alone in a porcine model of acute respiratory distress syndrome. Crit Care Med. 2000;28:2457-64.

30. Funk DJ, Graham MR, Girling LG, et al. A comparison of biologically variable ventilation to recruitment manoeuvres in a porcine model of acute lung injury. Respir Res. 2004;5:22.

31. Graham MR, Goertzen AL, Girling LG, et al. Quantitative computed tomography in porcine lung injury with variable versus conventional ventilation: recruitment and surfactant replacement. Crit Care Med. 2011;39:1721-30.

32. Thammanomai A, Hamakawa $H$, Bartolák-Suki $E$, Suki B. Combined effects of ventilation mode and positive end-expiratory pressure on mechanics, gas exchange and the epithelium in mice with acute lung injury. PLoS One. 2013;8:e53934

33. Nam AJ, Brower RG, Fessler HE, Simon BA. Biologic variability in mechanical ventilation rate and tidal volume does not improve oxygenation or lung mechanics in canine oleic acid lung injury. Am J Respir Crit Care Med. 2000;161:1797-804.

34. Graham MR, Gulati H, Kha L, Girling LG, Goertzen A, Mutch WAC. Resolution of pulmonary edema with variable mechanical ventilation in a porcine model of acute lung injury. Can J Anaesth. 2011;58:740-50.

35. Arold SP, Mora R, Lutchen KR, Ingenito EP, Suki B. Variable tidal volume ventilation improves lung mechanics and gas exchange in a rodent model of acute lung injury. Am J Respir Crit Care Med. 2002;165:366-71.

36. Spieth PM, Güldner A, Beda A, et al. Comparative effects of proportional assist and variable pressure support ventilation on lung function and damage in experimental lung injury. Crit Care Med. 2012;40:2654-61.
37. Spieth PM, Carvalho AR, Güldner A, et al. Effects of different levels of pressure support variability in experimental lung injury. Anesthesiology. 2009;110:342-50.

38. Arold SP, Suki B, Alencar AM, Lutchen KR, Ingenito EP. Variable ventilation induces endogenous surfactant release in normal guinea pigs. Am J Physiol Lung Cell Mol Physiol. 2003;285:L370-5.

39. Carvalho AR, Spieth PM, Güldner A, et al. Distribution of regional lung aeration and perfusion during conventional and noisy pressure support ventilation in experimental lung injury. J Appl Physiol. 2011;110(1985):1083-92.

40. Mutch WAC, Harms S, Graham RM, Kowalski SE, Girling LG, Lefevre GR. Biologically variable or naturally noisy mechanical ventilation recruits atelectatic lung. Am J Respir Crit Care Med. 2000;162:319-23.

41. Beda A, Güldner A, Simpson DM, et al. Effects of assisted and variable mechanical ventilation on cardiorespiratory interactions in anesthetized pigs. Physiol Meas. 2012;33:503-19.

42. Mutch WAC, Buchman TG, Girling LG, Walker EK-Y, McManus BM, Graham MR. Biologically variable ventilation improves gas exchange and respiratory mechanics in a model of severe bronchospasm. Crit Care Med. 2007;35:1749-55.

43. Rentzsch I, Santos CL, Huhle R, et al. Variable cell stretch reduces the release of CXCL-2 by LPS-stimulated L2 cells via the ERK1/2-pathway. Am J Respir Crit Care Med. 2015;191:A2042 (abst).

44. McMullen MC, Girling LG, Graham MR, Mutch WAC. Biologically variable ventilation improves oxygenation and respiratory mechanics during onelung ventilation. Anesthesiology. 2006;105:91-7.

45. Boker A, Haberman CJ, Girling L, et al. Variable ventilation improves perioperative lung function in patients undergoing abdominal aortic aneurysmectomy. Anesthesiology. 2004;100:608-16.

46. Kowalski S, McMullen MC, Girling LG, McCarthy BG. Biologically variable ventilation in patients with acute lung injury: a pilot study. Can J Anaesth. 2013;60:502-3.

47. Spieth PM, Güldner A, Uhlig C, et al. Variable versus conventional lung protective mechanical ventilation during open abdominal surgery: study protocol for a randomized controlled trial. Trials. 2014:15:155-5.

48. Spieth PM, Güldner A, Huhle R, et al. Short-term effects of noisy pressure support ventilation in patients with acute hypoxemic respiratory failure. Crit Care. 2013;17:R261

49. Kiss T, Güldner A, Bluth T, et al. Rationale and study design of ViPS - variable pressure support for weaning from mechanical ventilation: study protocol for an international multicenter randomized controlled open trial. Trials. 2013;14:363-3.

50. Güldner A, Beda A, Kiss T, et al. Effects of random and pseudo-random variable ventilation on lung function in experimental lung injury. Am J Respir Crit Care Med. 2012;185:A5442 (abst) 